\title{
Quantifying Poincare's Continuation Method for Nonlinear Oscillators
}

\author{
Daniel Núñez and Andrés Rivera \\ Pontificia Universidad Javeriana, Cali 760031, Colombia \\ Correspondence should be addressed to Daniel Núñez; denunez@javerianacali.edu.co
}

Received 16 June 2015; Accepted 5 August 2015

Academic Editor: Weinian Zhang

Copyright (C) 2015 D. Núñez and A. Rivera. This is an open access article distributed under the Creative Commons Attribution License, which permits unrestricted use, distribution, and reproduction in any medium, provided the original work is properly cited.

In the sixties, Loud obtained interesting results of continuation on periodic solutions in driven nonlinear oscillators with small parameter (Loud, 1964). In this paper Loud's results are extended out for periodically driven Duffing equations with odd symmetry quantifying the continuation parameter for a periodic odd solution which is elliptic and emanates from the equilibrium of the nonperturbed problem.

\section{Introduction}

After pioneering work of $\mathrm{H}$. Poincare in celestial mechanics, the continuation analytical method will have a great relevance in applied problems in science and technology. Several versions of this approach for the searching of dynamic objects like periodic solutions and invariant manifolds have been very fruitful in dynamical systems and its applications; see $[1,2]$. Perhaps there are perturbations of oscillators like

$$
\ddot{x}+g(x, \dot{x})=\epsilon p(t, x, \dot{x}, \epsilon),
$$

where $g, p$ are continuous and $p$ is $T$-periodic function in $t$ and $\epsilon$ is a small parameter; this is one of the easiest environments on which we can apply the continuation methods. In the sixties, Loud [3] obtained interesting results of local continuation in driven nonlinear differential equations like (1). He assumed that the nonperturbed equation

$$
\ddot{x}+g(x, \dot{x})=0
$$

had an isolated equilibrium $x \equiv 0$ and considered four cases according to the relative position of the Floquet multipliers of the variational equation

$$
\ddot{y}+b \dot{y}+a y=0,
$$

with $a=g_{x}(0,0), b=g_{\dot{x}}(0,0)$. We denote by $x\left(t, x_{0}, v_{0}\right)$ the general solution of $(*)$. Loud searched the solution of the implicit function system

$$
\begin{aligned}
& x\left(T, x_{0}, v_{0}, \epsilon\right)-x_{0}=0, \\
& \dot{x}\left(T, x_{0}, v_{0}, \epsilon\right)-x_{0}=0,
\end{aligned}
$$

in order to obtain $\epsilon$-family of $T$-periodic solutions as a continuation of the equilibrium $x \equiv 0$ for $\epsilon=0$. He used several versions of the implicit function theorem obtaining some orthogonality conditions involving the perturbation term $p(t, 0,0,0)$ and the solutions of the variational equation. According to a sign over this orthogonality condition it is possible to know the direction of movement of the Floquet multipliers while the parameter increases. In this way the author is able to classify the stability properties for $\epsilon$ small enough. For the frictionless and nonresonant case, that is,

$$
\begin{aligned}
& b=0, \\
& a \neq\left(\frac{2 n \pi}{T}\right)^{2}, \quad \forall n \in \mathbb{N}
\end{aligned}
$$

(as will be considered in this paper), Loud's result does not provide any stability information (see Theorem 2.9 in [3]). 
From now on, we are interested in studying the periodically driven Duffing equation

$$
\ddot{x}+g(x)=\epsilon p(t),
$$

with $g, p$ continuous, $p$ being $2 \pi$-periodic function, and $a=g^{\prime}(0)>0$ satisfying the above nonresonant condition. We assume that $x \equiv 0$ is an elliptic equilibrium for the nonperturbed problem $(\epsilon=0)$ and we formulate the following two questions:

(i) How small is the perturbation parameter $\epsilon$ to guarantee the linear stability for $(* *)$ ?

(ii) How small is the perturbation parameter $\epsilon$ to guarantee the nonlinear stability for $(* *)$ ?

A concrete example of oscillators like $(* *)$ is the forced pendulum

$$
\ddot{x}+\omega^{2} \sin x=\epsilon p(t),
$$

where many results with respect to the existence and stability of periodic solutions can be found in the literature [4-7]; see also [8] and the references therein. For instance, it is well known that if $p(t)$ is $2 \pi$-periodic function, there exists $2 \pi$ periodic solution for the forced pendulum as a continuation of the trivial solution which is stable when $\omega \notin \mathbb{N}_{0}$ and $\epsilon$ is small enough. This result is an easy consequence of the KAM theory.

There are at least three different types of analytical periodic continuation on the forced pendulum as follows: (i) the small oscillations previously mentioned, (ii) those emanating from certain periodic solutions of the nonlinear center for the nonforced case, and (iii) those emanating from the hanging solutions for the nonforced case [6]. In this last paper the author applied Loud's techniques in order to find suitable $p(t)$ to guarantee the bifurcations of many periodic solutions from the hanging one.

On the other hand, assuming appropriate symmetries on $(* *), g$ and $p$ odd functions, the implicit system $(L)$ could be reduced to a single scalar equation

$$
f\left(v_{0}, \epsilon\right)=x\left(\frac{T}{2}, 0, v_{0}, \epsilon\right)=0,
$$

in order to find odd $T$-periodic solutions. This is an original idea by Hamel [4] in his research of periodic solutions on the forced pendulum.

Thereby in this work we focus on the global continuation problem of periodic solutions under this kind of symmetries for $(* *)$ and their stability properties, starting from an elliptic equilibrium of the nonperturbed equation.

As the core problem has been reduced to an implicit one, that is, the study of the set of zeros of a continuous real function (e.g., the function $f$ ), some topological tools, like the Leray-Schauder Continuation Theorem [9], help us to understand its structure. This approach has been successfully applied in the study of periodic solutions on a restricted threebody problem (see $[10,11])$. In order to apply this tool it is necessary to compute a priori bounds over the zeros of $f$ (see Theorem 1 in Section 2 for more details) but the conclusion of the Leray-Schauder Theorem says nothing about the linear stability of the associated periodic solutions. For this study it is necessary to obtain more refined bounds over the periodic solution in order to apply some classical stability results on the variational equation (like Hill's equation).

The rest of the paper is divided in four sections. In Section 2 we illustrate how the Leray-Schauder Continuation Theorem can be applied to the forced pendulum in order to get a global family of periodic solutions from the equilibrium and remark its limitations for the stability analysis of this family. In Section 3 we consider oscillators of pendulum type with odd symmetries and present our first main result (Theorem 5); a family of odd periodic solution is obtained for all parameter values, and furthermore we present some interesting a posteriori bounds for its amplitude (see (25)). In Section 4 we review some basic facts about the stability of Hill's equation and we present the second main result, namely, the determination of a computable $\epsilon$-interval, where we guarantee the linear stability for the periodic continuation obtained in Theorem 5. Finally, Section 5 is devoted to point out some open questions about the nonlinear stability of the obtained periodic family.

\section{The Forced Pendulum and a Global Implicit Function Theorem}

Consider the forced pendulum

$$
\ddot{x}+\omega^{2} \sin x=\epsilon p(t),
$$

where $\epsilon$ is a positive parameter and $p(t)$ is an odd and $2 \pi$ periodic continuous function; that is, for all $t \in \mathbb{R}$, we have

$$
\begin{aligned}
p(t+2 \pi) & =p(t), \\
p(-t) & =-p(t) .
\end{aligned}
$$

The existence of odd and $2 \pi$-periodic solutions of (6) was proved for the first time by Hamel [4] in 1922 by means of a reduction to the boundary value problem

$$
\begin{aligned}
\ddot{x}+\omega^{2} \sin x & =\epsilon p(t), \\
x(0) & =x(\pi)=0 .
\end{aligned}
$$

See [8] for more references on this paradigmatic equation. Let $x(t, \eta, \epsilon)$ be the solution of (6) satisfying

$$
\begin{aligned}
& x(0)=0, \\
& \dot{x}(0)=\eta .
\end{aligned}
$$

This is a real analytic function in the arguments $(t, \eta, \epsilon) \in \mathbb{R}^{3}$ (see [12]) and is globally defined in $\mathbb{R}$. It is not difficult to prove that the research of odd and $2 \pi$-periodic solutions of (6) is equivalent to study (8). This follows by performing odd and $2 \pi$-periodic extensions improving the symmetries of (6) and its periodicity. So problem (8) can be reduced to the study of the implicit equation

$$
x(\pi, \eta, \epsilon)=0 .
$$


Therefore we want to apply some global version of the Implicit Function Theorem in order to solve (10), namely, the analytical version of the Leray-Schauder Continuation Theorem (see [9]), which provides parametrized curves $(\eta(s), \epsilon(s))$ solving (10) starting at $(\eta, \epsilon)=(0,0)$. We present the following version of this result. The complete proof can be found in [10].

First, we recall that for a given function $f \in C^{1}([a, b])$ which does not vanish in $\{a, b\}$ and has a finite number of nondegenerate zeros $x_{1}, \ldots, x_{n}$ in $] a, b$ [it is possible to define the Brouwer degree $\operatorname{deg}(f,[a, b])$ as

$$
\operatorname{deg}(f,[a, b])=\sum_{i=1}^{n} \operatorname{sign}\left(f^{\prime}\left(x_{i}\right)\right),
$$

where $f^{\prime}$ denotes the derivatives of $f$. If $x_{*}$ is an isolated zero in the set of zeros of $f$, the Brouwer index of the zero $x_{*}$ is defined by

$$
\text { ind }\left(f, x_{*}\right)=\operatorname{deg}(f, U) \text {, }
$$

where $U$ is a small neighbourhood of $x_{*}$. Now we are able to present the main theorem of this section and its application to $(10)$.

Theorem 1 (Leray-Schauder). Let $F: \mathbb{R} \times[a, b] \rightarrow \mathbb{R}$ be analytic and let $Z=\{(\eta, \epsilon): F(\eta, \epsilon)=0\}$ be the set of zeros of $F$. Assume the following:

(H1) $Z$ is bounded.

(H2) The set $Z_{a}=\left\{(\eta, a): F_{a}(\eta)=0\right\}$ with $F_{a}(\eta)=F(\eta, a)$ is finite and there is $\left(\eta_{0}, a\right) \in Z_{a}$ with ind $\left(F_{a}, \eta_{0}\right) \neq 0$.

Then there is a continuum $\operatorname{arc} \alpha:[0,1] \rightarrow Z, \alpha(s)=$ $(\eta(s), \epsilon(s))$, with $\eta(0)=\eta_{0}, \epsilon(0)=a$, such that either $\epsilon(1)=b$ or $\epsilon(1)=a$ and $\eta(1) \neq \eta_{0}$.

From Theorem 1 we have the following consequence for the forced pendulum.

Proposition 2. Given $\Delta>0, \omega \in] 0,1[$ there exists a continuum $\operatorname{arc} \eta=\eta(s), \epsilon=\epsilon(s), s \in[0,1]$ such that

$$
x_{s}(t)=x(t, \eta(s), \epsilon(s))
$$

is an odd $2 \pi$-periodic solution of forced pendulum (6) with $x(0)=0, \epsilon(0)=0$, and $\epsilon(1)=\Delta$.

Proof. Let $x(t, \eta, \epsilon)$ be the solution of (6) that satisfies initial conditions (9) and defines the real analytic function

$$
F(\eta, \epsilon)=x(\pi, \eta, \epsilon)
$$

with $\epsilon \in[0, \Delta]$. The set of zeros of $F$ is clearly bounded since the derivatives of the solutions of (6) are uniformly bounded in $[0,2 \pi]$ which reveals a simple integration over $(6)$ :

$$
|\dot{x}(t)| \leq|\epsilon|\|p\|_{1}+2 \pi \omega^{2}, \quad \forall t \in[0,2 \pi],
$$

where $\|\cdot\|_{1}$ denotes $L_{1}$-norm in the space $L^{1}([0,2 \pi])$; therefore

$$
|\eta| \leq \Delta\|p\|_{1}+2 \pi \omega^{2}
$$

then (H1) holds. On the other hand, since $\omega<1$, the only $2 \pi$ periodic solution for the nonforced pendulum $(\epsilon=0)$ is the trivial one. This nonlinear center is surrounded by periodic solutions $x(t, \eta, 0)$ with a monotone increasing time period function $T(\eta)$ with $\lim _{\eta \rightarrow 0} T(\eta)=2 \pi / \omega$ (see [13]); therefore if $\omega<1$ we obtain $T(\eta)>2 \pi$ for all $\eta \neq 0$. As a consequence the zeros of the function $F_{0}(\eta)=x(\pi, \eta, 0)$ are reduced to $\{(0,0)\}$.

Now we compute the index at $\eta_{0}=0$ by linearization; that is,

$$
\operatorname{ind}\left(F_{0}, \eta_{0}\right)=\operatorname{sign}\left(\frac{\partial F_{0}}{\partial \eta}(0,0)\right) \text {. }
$$

Notice that $\left(\partial F_{0} / \partial \eta\right)(0,0)=y(\pi)$ where $y(t)$ is the solution of the variational problem

$$
\begin{aligned}
\ddot{y}+\omega^{2} y & =0, \\
y(0) & =0, \\
\dot{y}(0) & =1 .
\end{aligned}
$$

Since $0<\omega<1$, then

$$
\operatorname{ind}\left(F_{0}, \eta_{0}\right)=\operatorname{sign}\left(\frac{\sin \omega \pi}{\omega}\right)=1,
$$

and this verifies $(\mathrm{H} 2)$. As a consequence, we infer the existence of a continuous family $\{(\eta(s), \epsilon(s))\}_{s \in[0,1]}$ in $\mathbb{R} \times[0, \Delta]$ such that

$$
\begin{aligned}
F(\eta(s), \epsilon(s)) & =0, \\
\eta(0) & =0, \\
\epsilon(0) & =0,
\end{aligned}
$$

and either

$$
e_{1}=\Delta
$$

or

$$
\begin{gathered}
e_{1}=0, \\
\eta(1) \neq \eta_{0},
\end{gathered}
$$

but this last alternative is not possible, again, because $\omega<1$. Then we get the required global continuation $x_{s}(t)$ of odd $2 \pi$ periodic solutions for (6).

Remark 3. Note that the continuation $x_{s}(t)$ can be identified with a parametrized curve in $\eta \epsilon$-plane and it could have turning points. See Figure 1. In the next section we will show that this is not the case and actually this curve is a graph of a differentiable function $\eta=\phi(\epsilon)$ globally defined on $\mathbb{R}^{+}$when $\omega \in[0,1 / 2[$.

Remark 4. Note that Leray-Schauder's Theorem demands $a$ priori bounds over the continuation $x_{s}(t)$ which are generally coarse. A key subject for researching of the stability of the continuation is the possibility of obtaining more acute 

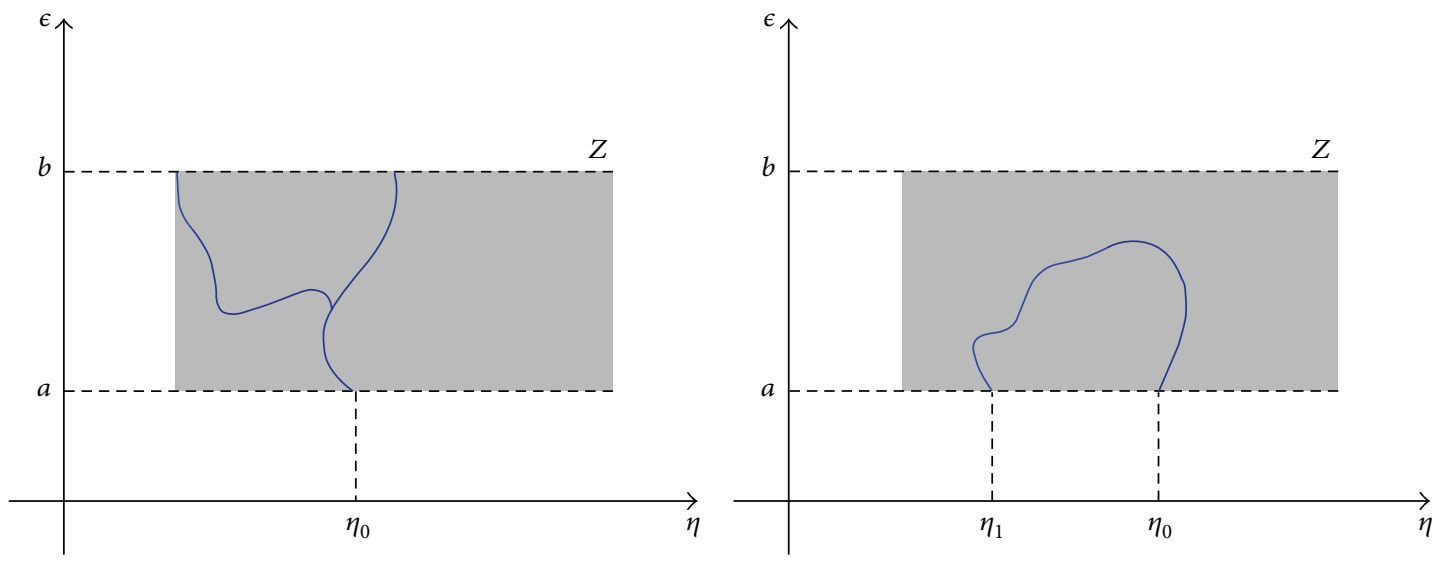

FIGURE 1: Graphic of the two possible options for the continuum arc which contains the point $\left(\eta_{0}, a\right)$ in the Leray-Schauder Continuation Theorem for real analytical functions.

bounds over it by means of some constructive approach. This is the purpose in the next section, to present some basic procedure for quantifying a linear stable branch of odd periodic solutions emerging from an equilibrium on oscillators of pendulum type.

\section{Odd Global Continuation of Equilibrium Solutions for Oscillators of Pendulum Type}

This section is devoted to oscillators of pendulum type given by the equation

$$
\ddot{x}+g(x)=\epsilon p(t),
$$

where $p$ is an odd $2 \pi$-periodic function and $g \in C^{\infty}(\mathbb{R})$ satisfies the following conditions for some positive numbers $\alpha_{0}$ and $\omega$ :

(1) $g(-x)=-g(x)$, for all $x \in \mathbb{R}$,

(2) $\left|g^{\prime}(x)\right| \leq \omega^{2}$, for all $x \in \mathbb{R}$,

(3) $g^{\prime}(x)>0$, for all $x \in\left[0, \alpha_{0}[\right.$,

(4) $g^{\prime}\left(\alpha_{0}\right)=0, g^{\prime \prime} \neq 0$ in $\left[0, \alpha_{0}[\right.$.

Without loss of generality, in the following we assume that

$$
\|p\|_{1}=\int_{0}^{2 \pi}|p(t)| d t=1 .
$$

The main result of this section is the following.

Theorem 5. If $\omega \in] 0,1 / 2]$, then there exist $x_{\epsilon}=x_{\epsilon}(t)$ being $2 \pi$-periodic and odd continuation of the equilibrium $x \equiv 0$ for (23), for all $\epsilon \in \mathbb{R}$. Moreover

$$
\left\|x_{\epsilon}\right\|_{\infty} \leq \frac{\sigma_{\omega}}{2 \omega}|\epsilon|\left(1+\frac{\sinh \omega \pi}{\sin \omega \pi}\right)
$$

with

$$
\sigma_{\omega}=\max \left\{\sinh \omega \pi, \frac{\sinh 2 \omega \pi-\sin 2 \omega \pi}{2}\right\}
$$

Remark 6. As a particular case, this theorem gives the odd global continuation of the equilibrium solution $x \equiv 0$ for forced pendulum (6) for all frequencies $\omega \in] 0,1 / 2]$.

The proof of Theorem 5 requires several preliminary results. We start with an estimative over the canonical solutions of Hill's equation as a consequence of Sturm's Comparison Theorem.

Lemma 7. Let $a \in C([0, T / 2])$ with $|a(t)| \leq \omega^{2}$ for all $t \in$ $[0, T / 2]$ and $|a| \neq \omega^{2}$ with $\omega \in[0, \pi / T]$. Let $\phi_{1}(t)$ and $\phi_{2}(t)$ be the solutions of

$$
\ddot{x}+a(t) x=0,
$$

satisfying the initial conditions

$$
\begin{gathered}
\phi_{1}(0)=1, \\
\dot{\phi}(0)=0, \\
\phi_{2}(0)=0, \\
\dot{\phi}_{2}(0)=1,
\end{gathered}
$$

respectively. Then for all $t \in] 0, T / 2[$ we have the following inequalities:

$$
\begin{aligned}
\cos \omega t & <\phi_{1}(t)<\cosh \omega t, \\
\frac{1}{\omega} \sin \omega t & <\phi_{2}(t)<\frac{1}{\omega} \sinh \omega t .
\end{aligned}
$$

Proof. Let $\mu_{i}(t), i=1,2$ be the canonical solutions of

$$
\ddot{x}+\omega^{2} x=0
$$

that satisfy the initial conditions $\mu_{1}(0)=\dot{\mu}_{2}(0)=1$ and $\mu_{2}(0)=\dot{\mu}_{1}(0)=0$; that is,

$$
\begin{aligned}
& \mu_{1}(t)=\cos \omega t \\
& \mu_{2}(t)=\frac{1}{\omega} \sin \omega t .
\end{aligned}
$$


For $i=1,2$ observe that $\chi_{i}=\phi_{i}-\mu_{i}$ satisfies the boundary problem

$$
\begin{aligned}
\ddot{x}+a(t) x+q_{i}(t) & =0, \\
x(0) & =0, \\
\dot{x}(0) & =0,
\end{aligned}
$$

with $q_{i}(t)=\left(a(t)-\omega^{2}\right) \mu_{i}$. So it is not difficult to prove that

$$
\chi_{i}(t)=\int_{0}^{t} G(t, s) q_{i}(s) d s
$$

where

$$
G(t, s)=\phi_{1}(t) \phi_{2}(s)-\phi_{1}(s) \phi_{2}(t) .
$$

Now we want to know the sign of $\chi_{i}(t)$ on [0,T/2]. First, we remark that $q_{i}(t) \leq 0$ since $a(t)-\omega^{2} \leq 0$ and $\mu_{i} \geq 0$ for all $[0, T / 2[$ because $\omega \leq \pi / T$.

On the other hand, since $a(t) \leq(\pi / T)^{2}$ on $[0, T / 2]$, from the Sturm Comparison Theorem, we deduce $\phi_{2}(t)>0$ in ] $0, T / 2]$. This implies that $G(t, s)<0$ for all $(t, s) \in\{(t, s): 0<$ $s<t, 0<t<T / 2\}$ (see [14]). From the hypothesis on $a(t)$ we know that $q_{i}(t)$ is not identically zero; then as a consequence we have $\chi_{i}(t)>0$ on $\left.] 0, T / 2\right]$; that is,

$$
\begin{aligned}
\cos \omega t & <\phi_{1}(t), \\
\frac{1}{\omega} \sin \omega t & <\phi_{2}(t), \\
\forall t & \left.\in] 0, \frac{T}{2}\right] .
\end{aligned}
$$

The proof for the other inequalities follows the same ideas if we consider the equation

$$
\ddot{x}-\omega^{2} x=0
$$

and the canonical solutions

$$
\begin{aligned}
& \tilde{\mu}_{1}(t)=\cosh \omega t, \\
& \tilde{\mu}_{2}(t)=\frac{1}{\omega} \sinh \omega t .
\end{aligned}
$$

As in the previous section, let $x(t, \eta, \epsilon)$ be the solution of (23) satisfying

$$
\begin{aligned}
& x(0)=0, \\
& \dot{x}(0)=\eta .
\end{aligned}
$$

From the symmetries over (23) the search of odd $2 \pi$-periodic solutions can be focused to solve the Dirichlet problem (in a half period)

$$
\begin{aligned}
\ddot{x}+g(x) & =\epsilon p(t), \\
x(0) & =0, \\
x(\pi) & =0 .
\end{aligned}
$$

In other words, we must find the zeros of the function

$$
x(\pi, \eta, \epsilon)=0 .
$$

This is a classical implicit function problem with initial solution of the trivial one $\eta=\epsilon=0$. Let $\omega_{0}^{2}:=g^{\prime}(0)$. Since $\omega_{0}<\omega<1 / 2$, then

$$
\partial_{\eta} x(\pi, 0,0)=\frac{1}{\omega_{0}} \sin \left(\omega_{0} \pi\right) \neq 0
$$

as a consequence there exists a local function $\eta=\eta(\epsilon)$ such that $x(t, \eta(\epsilon), \epsilon)=0$. Let $\mathscr{V}$ be an open neighborhood of $(0,0)$ where $\partial_{\eta} x(\pi, \eta, \epsilon) \neq 0$. By the Implicit Function Theorem problem (40) is equivalent to the Cauchy Problem

$$
\begin{aligned}
\eta^{\prime}(\epsilon) & =F(\eta, \epsilon), \\
\eta(0) & =0,
\end{aligned}
$$

which is well defined on $\mathscr{V}$ with

$$
F(\eta, \epsilon)=-\frac{\partial_{\epsilon} x(\pi, \eta, \epsilon)}{\partial_{\eta} x(\pi, \eta, \epsilon)} .
$$

The next proposition gives sufficient conditions to guarantee that $\mathscr{V}=\mathbb{R}^{2}$ and $F$ is bounded.

Proposition 8. If $\omega \in] 0,1 / 2]$, then the function $F(\eta, \epsilon)$ given in (43) is well defined in all $\mathbb{R}^{2}$ and

$$
|F(\eta, \epsilon)| \leq \frac{\sigma_{\omega}}{2 \sin \pi \omega},
$$

with $\sigma_{\omega}=\max \{\sinh \pi \omega,(\sinh 2 \pi \omega-\sin 2 \pi \omega) / 2\}$.

Proof. For each $(\eta, \epsilon) \in \mathbb{R}^{2}$ consider the variational equation

$$
\begin{aligned}
\ddot{x}+a(t) x & =0, \\
a(t) & =a_{\eta, \epsilon}(t)=g^{\prime}(x(t, \eta, \epsilon)) .
\end{aligned}
$$

From assumption (2) over the function $g$, it follows that $|a| \leq \omega^{2}$ in $[0, \pi]$ uniformly in $(\eta, \epsilon)$ and for assumption (3) it follows that $a \neq \omega^{2}$. Let $\phi_{1}$ and $\phi_{2}$ be the canonical solutions of (45). From Lemma 7 with $T=2 \pi$ we obtain

$$
\begin{aligned}
\cos \omega t & <\phi_{1}(t)<\cosh \omega t, \\
\frac{1}{\omega} \sin \omega t & <\phi_{2}(t)<\frac{1}{\omega} \sinh \omega t,
\end{aligned}
$$

for all $t \in] 0, \pi]$. On the other hand, the theorem of differentiability with respect to initial conditions and parameters implies that the functions $\phi_{2}(t)=\partial_{\eta} x(t, \eta, \epsilon)$ and $\psi(t)=$ $\partial_{\epsilon} x(t, \eta, \epsilon)$ satisfy the following Cauchy problems:

$$
\begin{aligned}
\ddot{x}+a(t) x & =0, \\
x(0) & =\dot{x}(0)=1, \\
\ddot{x}+a(t) x & =p(t), \\
x(0) & =\dot{x}(0)=0,
\end{aligned}
$$


respectively. Therefore, for $t \in[0, \pi]$, we have

$$
\psi(t)=-\int_{0}^{t} G(t, s) p(s) d s
$$

Since $\omega \in] 0,1 / 2[$ from the Comparison Sturm Theorem we obtain $\phi_{2}(t)>0$ for all $\left.t \in\right] 0, \pi[$. Following the lemma in [14] we concluded that

$$
G(t, s)=\phi_{1}(t) \phi_{2}(s)-\phi_{1}(s) \phi_{2}(t)<0,
$$

for all $(t, s) \in \Delta=\{(t, s) \mid 0<s<t, 0<t<\pi\}$. Moreover since $0<\omega \pi<\pi / 2$ then $\cos \omega t>0$ and $\sin \omega t>0$ on $[0, \pi]$; therefore

$$
|G(t, s)|=-G(t, s) \leq \max _{(t, s) \in \bar{\Delta}} H(t, s)
$$

where $H(t, s)=(1 / \omega)(\cosh \omega t \sinh \omega s-\cos \omega s \sin \omega t)$. Since there are no critical points of $H$ in $\Delta$ then $H$ has the maximum value on the boundary of $\Delta$. A simple calculation shows that

$$
|G(t, s)|=-G(t, s) \leq \frac{\sigma_{\omega}}{\omega}
$$

with

$$
\sigma_{\omega}=\max \left\{\sinh \pi \omega, \frac{\sinh 2 \pi \omega-\sin 2 \pi \omega}{2}\right\}
$$

On the other hand

$$
|\psi(t)| \leq \frac{\sigma_{\omega}}{2 \omega} \quad \forall t \in[0, \pi] .
$$

Finally we obtain

$$
|F(\eta, \epsilon)|=\left|\frac{\psi(\pi)}{\phi_{2}(\pi)}\right| \leq \frac{\sigma_{\omega}}{2 \sin \pi \omega} .
$$

Remark 9. For the autonomous Cauchy problem

$$
\begin{aligned}
\frac{d \eta}{d \epsilon} & =F(\eta, t), \\
\frac{d t}{d \epsilon} & =1, \\
\eta(0) & =0, \\
t(0) & =0,
\end{aligned}
$$

which has a bounded vector field $X(\eta, \epsilon)=(F(\eta, \epsilon), 1)$; we conclude that $\eta(\epsilon)$ is well defined in ] - $\infty, \infty[$ (see [15]); therefore for all $\omega \in] 0,1 / 2]$ we obtain an odd, $2 \pi$-periodic continuation $\varphi_{\epsilon}(t)=x(t, 0, \eta(\epsilon), \epsilon)$ of the equilibrium for all $\epsilon \in \mathbb{R}$.

Now we are able to present the proof of the main theorem of this section.
Proof of Theorem 5. Let $X(\epsilon)=(\eta(\epsilon), t(\epsilon))^{\mathrm{tr}}$ and consider the autonomous Cauchy problem

$$
\begin{aligned}
X^{\prime} & =\mathscr{F}(X)=(F(\eta, \epsilon), 1), \\
X(0) & =(0,0) .
\end{aligned}
$$

It follows from Proposition 8 that $\mathscr{F}$ is bounded. From here we deduce that all solutions are globally defined; then $\eta(\epsilon)$ is well defined for all $t \in]-\infty, \infty[$; (see [15]). As a consequence for all $\omega \in] 0,1 / 2]$ we obtain an odd, $2 \pi$-periodic continuation $\varphi_{\epsilon}(t)=x(t, 0, \eta(\epsilon), \epsilon)$ of the equilibrium for all $\epsilon \in \mathbb{R}$. Now by the Mean Value Theorem we have

$$
\left|\varphi_{\epsilon}\right| \leq|\epsilon| \max _{|\xi| \leq \epsilon, t \in[0, \pi]}\left|\partial_{\epsilon} \varphi_{\epsilon}(t, \eta(\xi), \xi)\right|
$$

On the other hand,

$$
\left|\partial_{\epsilon} \varphi_{\epsilon}\right| \leq\left|\partial_{\eta} x\right|\left|\eta^{\prime}\right|+\left|\partial_{\epsilon} x\right|
$$

Since $\partial_{\epsilon} x$ and $\partial_{\eta} x$ are, respectively, the solutions of the initial value problems (47) and (48), with $a(t)=g^{\prime}\left(\varphi_{\epsilon}(t)\right)$, they satisfy inequalities (46) and (54) in $[0, \pi]$; therefore

$$
\begin{aligned}
\left|\partial_{\epsilon} \varphi\right| & \leq \frac{\sigma_{\omega}}{2 \sin \pi \omega} \frac{\sinh \omega \pi}{\omega}+\frac{\sigma_{\omega}}{2 \omega} \\
& =\frac{\sigma_{\omega}}{2 \omega}\left(1+\frac{\sinh \omega \pi}{\sin \omega \pi}\right) ;
\end{aligned}
$$

as a consequence

$$
\left|\varphi_{\epsilon}\right| \leq|\epsilon| \frac{\sigma_{\omega}}{2 \omega}\left(1+\frac{\sinh \omega \pi}{\sin \omega \pi}\right)
$$

concluding the proof.

\section{Linear Stability of the Continuation}

In this section we will obtain a proof of the linear stability of the continuation $\varphi_{\epsilon}$. We use techniques that are traditionally employed in the study of Hill's equation. More precisely, the proof will be based on the Lyapunov-Zukovskii stability criteria for Hill's equations. We start with a well-known result of the Sturm comparison theory for Hill's equation.

Lemma 10. If $a \in C_{T}(\mathbb{R})$ satisfies $0<m^{2} \leq a(t) \leq M^{2}$ on $[0, T]$ with strict inequality on a set of positive measures on $[0, T]$, then

(i) any solution of (27) has infinitely many zeros;

(ii) if $t_{1}$ and $t_{2}$ are two consecutive zeros of any solution of (27), they satisfy

$$
\frac{\pi}{M}<t_{2}-t_{1}<\frac{\pi}{m}
$$

The proof of Lemma 10 follows easily from Sturm's Comparison Theorems (see [16]). 
Proposition 11 (Lyapunov-Zukovskii). Let $a \in C_{T}(\mathbb{R})$ such that $0<a(t) \leq(\pi / T)^{2}$, for all $t \in \mathbb{R}$, with strict inequality on a set of positive measures in $[0, T]$. Then (27) is elliptic.

Proof. By a contradiction argument suppose that there is a real Floquet multiplier $\lambda$. Let $\varphi(t)$ be the Floquet solution associated with $\lambda$; then

$$
\varphi(t+T)=\lambda \varphi(t), \quad \forall t \in \mathbb{R}
$$

Since $0<a(t)$ it follows from Lemma 10 that $\exists t_{0} \in \mathbb{R}$ such that $\varphi\left(t_{0}\right)=0$; then $\varphi\left(t_{0}+T\right)=0$. Once again, from Lemma $10 t_{0}$ and $t_{0}+T$ are consecutive zeros; as a consequence the distance is at most $T$ and this is a contradiction.

Finally we completed all the necessary arguments that we need in order to prove our second main result.

Theorem 12. Under the same hypothesis of Theorem $5,2 \pi$ periodic continuation $\varphi_{\epsilon}$ of the equilibrium $x=0$ of (45) is elliptic if $|\epsilon|<I=I(\omega)$ where

$$
I(\omega)=\frac{2 \alpha_{0} \omega \sin \pi \omega}{\sigma_{\omega}(\sinh \pi \omega+\sin \pi \omega)} .
$$

Proof. With $\epsilon \in]-I, I$ [ and from (25) it is clear that $\left|\varphi_{\epsilon}(t)\right| \leq$ $\alpha_{0}$. On the other hand, from the conditions on the function $g$, we have

$$
0<a(t)=g^{\prime}\left(\varphi_{\epsilon}(t)\right) \leq \omega^{2} \leq \frac{1}{4}, \quad \forall t \in \mathbb{R}
$$

On the other hand, if $a(t)=1 / 4$ for all $t \in \mathbb{R}$, then $\varphi_{\epsilon} \equiv 0$ since $g^{\prime}$ is strict monotone in ]0, $\alpha_{0}$ ] and this implies $p \equiv 0$ which is a contradiction. By continuity we deduce the existence of a set of positive measures in $[0, T]$ such that $0<a<$ $1 / 4$. The conclusion follows if we apply Proposition 11 to the variational equation along $\varphi_{\epsilon}$ :

$$
\ddot{y}+a(t) y=0,
$$

where $a(t)=g^{\prime}\left(\varphi_{\epsilon}(t)\right)$.

Corollary 13. For $p \in C_{2 \pi}(\mathbb{R}),\|p\|_{1}=1$, and odd $\omega \in$ ] $0,1 / 2]$, the equilibrium solution $x \equiv 0$ of the oscillator (23) can be continued like an odd $2 \pi$-periodic solution $\varphi_{\epsilon}(t)$ of the forced pendulum

$$
x^{\prime \prime}+\omega^{2} \sin x=\operatorname{\epsilon p}(t)
$$

which is elliptic (therefore linear stable) for $|\epsilon|<\gamma(\omega)$ where

$$
\gamma(\omega)=\frac{\pi \omega \sin \pi \omega}{\sigma_{\omega}(\sinh \pi \omega+\sin \pi \omega)} ;
$$

moreover,

$$
\left\|\varphi_{\epsilon}\right\| \leq \frac{|\epsilon| \sigma_{\omega}}{2 \omega}\left(1+\frac{\sinh \pi \omega}{\sin \pi \omega}\right)
$$

\section{Odd Global Continuation for any Positive $\omega$}

In order to remove the restriction $\omega \in] 0,1 / 2[$ in Theorem 5, we follow a different approach to estimate an upper bound for $\left|\partial_{\epsilon} x(\pi ; \eta, \epsilon)\right|$ and a lower bound for $\left|\partial_{\eta} x(\pi ; \eta, \epsilon)\right|$ in (42)-(43). As before we assume the condition $\|p\|_{1}=1$ on oscillator (23).

We start this new approach by setting new conditions over the function $g$. Suppose that there exist positive values $\omega$ and $\omega_{0} \notin \mathbb{N}$ such that
(a) $\left|g^{\prime}(x)\right|,\left|g^{\prime \prime}(x)\right| \leq \omega^{2}$, for all $x \in \mathbb{R}$.
(b) $g^{\prime}(0)=\omega_{0}^{2}$.

The main result of this section is the following.

Theorem 14. Under assumptions (a) and (b), there exist $\varphi_{\epsilon}=$ $\varphi_{\epsilon}(t)$ being $2 \pi$-periodic and odd continuation of the equilibrium $x \equiv 0$ for (23) for all $|\epsilon|<\Delta_{0}$ with

$$
\Delta_{0}=\Delta_{0}\left(\omega, \omega_{0}\right)=\frac{\left(1+\omega^{-2}\right)}{\omega_{0} A_{\omega}^{3}\left(A_{\omega}^{2}-1\right)}\left|\sin \omega_{0} \pi\right| .
$$

Moreover

$$
\left|\varphi_{\epsilon}(t)\right| \leq|\epsilon| A_{\omega}\left(1+\frac{A_{\omega}}{\Upsilon\left(\omega, \omega_{0}, \epsilon\right)}\right),
$$

where $A_{\omega}^{2}=e^{\left(1+\omega^{2}\right) \pi}$ and $\Upsilon\left(\omega, \omega_{0}, \epsilon\right)$ is a positive constant for $|\epsilon|<\Delta_{0}$ given by

$$
\Upsilon\left(\omega, \omega_{0}, \epsilon\right)=\left|\frac{1}{\omega_{0}} \sin \omega_{0} \pi\right|-\frac{|\epsilon| \omega^{2} A_{\omega}^{3}}{1+\omega^{2}}\left(A_{\omega}^{2}-1\right) .
$$

The proof of Theorem 14 follows similar arguments and steps are given in Section 2 for proving Theorem 5 . We start by considering again the solutions $\phi_{2}(t)=\partial_{\eta} x(t, \eta, \epsilon)$ and $\psi(t)=\partial_{\epsilon} x(t, \eta, \epsilon)$ of Cauchy problems (47) and (48), respectively.

Before the proof of Theorem 14 we point out some preliminary results. The first one is about the growing of the solutions for Hill's equations.

Lemma 15. Let $a \in C([0, T])$ with $|a(t)| \leq \sigma$ for all $t \in[0, T]$ and let $\phi_{1}(t), \phi_{2}(t)$ be the unique solutions of

$$
\ddot{x}+a(t) x=0
$$

satisfying

$$
\begin{aligned}
& \phi_{1}(0)=\dot{\phi}_{2}(0)=1, \\
& \dot{\phi}_{1}(0)=\phi_{2}(0)=0 .
\end{aligned}
$$

Then, for $i=1,2$, we have

$$
\left|\phi_{i}(t)\right| \leq e^{((1+\sigma) / 2) T} \quad \forall t \in[0, T] .
$$

Proof. Let $x(t)$ be a solution of $(\diamond)$ and define the function

$$
\mathscr{V}(t)=\frac{1}{2}\left(x^{2}(t)+\dot{x}^{2}(t)\right) .
$$


By a direct computation we obtain

$$
\begin{aligned}
\dot{\mathscr{V}}(t) & =x \dot{x}(1-a(t)) \leq \frac{\left(x^{2}+\dot{x}^{2}\right)}{2}(1+\sigma) \\
& =\mathscr{V}(t)(1+\sigma) .
\end{aligned}
$$

Solving this differential inequality with initial condition $\mathscr{V}(0)$ we get

$$
\mathscr{V}(t) \leq \mathscr{V}(0) e^{(1+\sigma) t} .
$$

In particular for the canonical solutions $\phi_{i}(t) i=1,2$ we have $\mathscr{V}(0)=1 / 2$ and therefore

$$
\left|\phi_{i}(t)\right| \leq\left\|\left(\phi_{i}(t), \dot{\phi}_{i}(t)\right)\right\| \leq e^{((1+\sigma) / 2) t}, \quad \forall t \in[0, T] .
$$

From (49), hypothesis (a), and Lemma 15 taking $a(t)=$ $g^{\prime}(x(t, \eta, \epsilon))$ on $[0, \pi]$ we have the following estimative:

$$
\left|\partial_{\epsilon} x(\pi, \eta, \epsilon)\right| \leq e^{\left(1+\omega^{2}\right) \pi}, \quad \forall(\eta, \epsilon) \in \mathbb{R}^{2} .
$$

The second preliminary result is the classical fundamental inequality in ordinary differential equations (see [16]).

Proposition 16. Suppose that $x(t), y(t)$ satisfy

$$
\begin{aligned}
\dot{x} & =f(t, x), \\
x\left(t_{0}\right) & =x_{0}, \\
\dot{y} & =g(t, y), \\
y\left(t_{0}\right) & =y_{0},
\end{aligned}
$$

where $f, g: U \subset \mathbb{R} \times \mathbb{R}^{n} \rightarrow \mathbb{R}^{n}$, are continuous and Lipschitz with respect to the second variable with Lipschitz constant $K$. If

$$
|f(t, u)-g(t, u)| \leq \delta, \quad(t, u) \in U
$$

then

$$
|x(t)-y(t)| \leq\left|x_{0}-y_{0}\right| e^{K\left|t-t_{0}\right|}+\frac{\delta}{K}\left(e^{K\left|t-t_{0}\right|}-1\right),
$$

for all $t$ in a common interval of existence.

Proof of Theorem 14. The existence of nontrivial odd, $2 \pi$ periodic solutions of (23) is equivalent to the solving of problem (40). Furthermore, from assumption (b)

$$
\partial_{\eta} x(\pi, 0,0)=\frac{1}{\omega_{0}} \sin \left(\omega_{0} \pi\right) \neq 0
$$

therefore we can consider the Cauchy problem

$$
\begin{aligned}
& \eta^{\prime}(\epsilon)=-\frac{\partial_{\epsilon} x(\pi, \eta, \epsilon)}{\partial_{\eta} x(\pi, \eta, \epsilon)}, \\
& \eta(0)=0
\end{aligned}
$$

in a small rectangle of the form $\Omega_{\Delta, \Gamma}=\{(\epsilon, \eta):|\epsilon| \leq \Delta,|\eta| \leq$ $\Gamma\}$.
Our objective is to solve initial value problem (84) in a concrete $\epsilon$-interval and on an appropriate rectangle. For this purpose we will estimate an upper bound over the absolute value of the right hand side of (84). In accordance with (79) we only have to find a positive lower bound for $\left|\partial_{\eta} x(t, \eta, \epsilon)\right|$ on $\Omega_{\Delta, \Gamma}$.

Consider variational equation (45) given by

$$
\begin{aligned}
\ddot{x}+a_{\eta, \epsilon}(t) x & =0, \\
a_{\eta, \epsilon}(t) & =g^{\prime}(x(t, \eta, \epsilon)) .
\end{aligned}
$$

The vector field of the first order system associated with this variational equation is given by

$$
F_{\eta, \epsilon}(t, x, y)=\left(y,-a_{\eta, \epsilon}(t) x\right) \text {, }
$$

with $y=x^{\prime}$. Notice that this vector field is continuous and $(x, y)$-Lipschitz, with Lipschitz constant $K=1+\omega^{2}$ uniformly in $(\epsilon, \eta) \in \mathbb{R}^{2}$.

Next we apply the fundamental inequality to the vector fields $F_{\eta, \epsilon}(t, x, y), F_{0,0}(t, x, y)$ and the respective solutions $\partial_{\eta} x(t, \eta, \epsilon), \partial_{\eta} x(t, 0,0)=\left(1 / \omega_{0}\right) \sin \left(\omega_{0} t\right)$ (both in the same initial conditions) on the domain $U=\mathbb{R} \times]-A_{\omega}, A_{\omega}[\times \mathbb{R}$, where $A_{\omega}=e^{\left(\left(1+\omega^{2}\right) / 2\right) \pi}$ is an upper bound for the canonical solutions of (45) given by Lemma 15 . Henceforth

$$
\left|\partial_{\eta} x(\pi, \eta, \epsilon)-\frac{1}{\omega_{0}} \sin \omega_{0} \pi\right| \leq \frac{\delta}{1+\omega^{2}}\left(A_{\omega}^{2}-1\right),
$$

where $\delta$ is an upper bound on $U$ for

$$
\left|F_{\eta, \epsilon}(t, x, y)-F_{0,0}(t, x, y)\right|=\left|\left(a_{\eta, \epsilon}(t)-\omega_{0}^{2}\right) x\right| \text {. }
$$

From the mean value theorem we get

$$
\left|a_{\eta, \epsilon}(t)-\omega_{0}^{2}\right| \leq|\epsilon| m,
$$

where $m$ is any positive number such that

$$
m \geq \sup \left\{\left|\frac{\partial a_{\eta, \epsilon}(t)}{\partial \epsilon}\right|: t \in[0, \pi], \eta \in \mathbb{R}, \epsilon>0\right\} .
$$

Besides,

$$
\left|\frac{\partial a_{\eta, \epsilon}(t)}{\partial \epsilon}\right|=\left|g^{\prime}(x(t, \eta, \epsilon))\right|\left|\partial_{\epsilon} x(t, \eta, \epsilon)\right|,
$$

and from (79) and condition (a) over the function $g$ we have

$$
\left|\frac{\partial a_{\eta, \epsilon}(t)}{\partial \epsilon}\right| \leq \omega^{2} A_{\omega}^{2}
$$

for all $t \in[0, \pi], \eta \in \mathbb{R}, \epsilon>0$. In conclusion on the domain $U$ we have

$$
\begin{aligned}
& \left|F_{\eta, \epsilon}(t, x, y)-F_{0,0}(t, x, y)\right|=\left|\left(a_{\eta, \epsilon}(t)-\omega_{0}^{2}\right) x\right| \leq \delta \\
& \quad:=|\epsilon| \omega^{2} A_{\omega}^{3} .
\end{aligned}
$$


Now we impose the restriction (see (87))

$$
\frac{\delta}{1+\omega^{2}}\left(A_{\omega}^{2}-1\right)<\left|\frac{1}{\omega_{0}} \sin \omega_{0} \pi\right| .
$$

The restriction ( $\mathbf{R}$ ) is equivalent to $|\epsilon|<\Delta_{0}$ with $\Delta_{0}$ given by (70). Combining (87) and (R) we obtain (for all $u, v, \gamma \in \mathbb{R}$ if $|u-v|<\gamma<|v|$, then $|u|>|v|-\gamma)$

$$
\left|\partial_{\eta} x(\pi, \eta, \epsilon)\right| \geq \Upsilon\left(\omega, \omega_{0}, \epsilon\right)>0
$$

with $\Upsilon\left(\omega, \omega_{0}, \epsilon\right)$ given by (72). Finally, an upper bound for the modulus of the right hand side in (84) is given by

$$
\left|\frac{\partial_{\epsilon} x(\pi, \eta, \epsilon)}{\partial_{\eta} x(\pi, \eta, \epsilon)}\right| \leq M:=\frac{A_{\omega}^{2}}{\Upsilon\left(\omega, \omega_{0}, \epsilon\right)}
$$

on $\Omega_{\Delta_{0}, \Gamma}$. For classical Peano's Theorem we obtain a solution $\eta=\phi(\epsilon)$ of $(10)$ for $|\epsilon| \leq \min \left\{\Delta_{0}, \Gamma / M\right\}$. Note that our estimatives do not depend on $\eta$; therefore we can take $\Gamma \geq M \Delta_{0}$ so the solution $\phi(\epsilon)$ is defined on $]-\Delta_{0}, \Delta_{0}$ [. So we obtain the odd $2 \pi$-periodic family $\varphi_{\epsilon}(t)=x(t, \phi(\epsilon), \epsilon)$. Following the lines in the proof of Theorem 5 (inequalities (58) and (59)) is a straightforward task to obtain (71) using the above estimative.

\section{Concluding Remarks}

Applying the techniques in [7] to forced pendulum (6) we obtain a finite $\epsilon$-interval for frequencies $\omega$ far away of the strong resonances. In contrast, our first main result (Theorem 5) provides an infinite $\epsilon$-interval for low frequencies $\omega \in[0,1 / 2]$. Moreover for high frequencies we obtain $2 \pi$ periodic continuation of the equilibrium $x \equiv 0$ in a quantified $\epsilon$-interval (Theorem 14) for general oscillators of pendulum type.

The nonlinear stability in the Lyapunov sense of the family $\varphi_{\epsilon}$ obtained before remains an open problem. This can be studied by means of the KAM Theory. More precisely, the Twist Theorem of Möser [17] gives sufficient conditions to ensure that Poincare's mapping $\mathscr{P}_{\epsilon}$ of (23) has invariant curves in all neighbourhood of the elliptic fixed point $z_{0}=$ $\left(\varphi_{\epsilon}(0), \dot{\varphi}_{\epsilon}(0)\right)$.

From Möser's work this approach has been referenced in the literature like a third-order method, because the stability is generically decided by the nonvanishing of a certain thirdorder coefficient $\beta$ of $\mathscr{P}_{\epsilon}$ called the first twist coefficient. The Möser Theorem essentially claims that if $\beta \neq 0$, then the fixed point $z_{0}$ is stable and therefore $\varphi_{\epsilon}$ is also stable. We will explain this a little bit more.

By means of a translation $\varphi_{\epsilon}$ to the origin $y=x-\varphi_{\epsilon}$ and making Taylor's expansion up to the third order, we arrive to the equivalent equation with equilibrium $y \equiv 0$ :

$$
\ddot{y}+a_{\epsilon}(t) y+b_{\epsilon}(t) y+c_{\epsilon}(t) y^{3}+\cdots=0,
$$

where $a_{\epsilon}, b_{\epsilon}$, and $c_{\epsilon}$ and the remaining terms are periodic functions of period $2 \pi$; moreover

$$
\begin{aligned}
& a_{\epsilon}(t)=g^{\prime}\left(\varphi_{\epsilon}(t)\right), \\
& b_{\epsilon}(t)=\frac{g^{\prime \prime}\left(\varphi_{\epsilon}(t)\right)}{2}, \\
& c_{\epsilon}(t)=\frac{g^{\prime \prime \prime}\left(\varphi_{\epsilon}(t)\right)}{6} .
\end{aligned}
$$

The twist coefficient $\beta=\beta(\epsilon)$ is a meromorphic function which depends on $a_{\epsilon}, b_{\epsilon}$, and $c_{\epsilon}$ in a not easy way (see $[7,14,18$, 19]). In [14] Ortega gave for the first time an explicit formula for $\beta$ and subsequently Zhang in $[7,19]$ provided some refinements of it making the dependence with respect to $a_{\epsilon}$ more synthetic and very practical for applications (see $[5,7,19-21])$. The open problem consists of studying the first zero of $\beta(\epsilon)$ in order to estimate $\epsilon$-range for the stability of $\varphi_{\epsilon}$. In this direction the results in [7] could be useful. Finally, it is important to notice that when $g$ is really analytical the recently Hanßmann and Simó's results in [22] could be useful to study the nonlinear stability for the continuation.

\section{Conflict of Interests}

The authors declare that there is no conflict of interests regarding the publication of this paper.

\section{Acknowledgments}

This work was supported by Capital Semilla (2014-2015), Project 00003967, and Pontificia Universidad Javeriana, Seccional Cali, Cali, Colombia.

\section{References}

[1] P. Gomis-Porqueras and À. Haro, "A geometric description of a macroeconomic model with a center manifold," Journal of Economic Dynamics and Control, vol. 33, no. 6, pp. 1217-1235, 2009.

[2] C. Simó, "On the analytical and numerical approximation of invariant manifolds," in Les Méthodes Modernes de la Mecánique Céleste, D. Benest and C. Froeschlé, Eds., Course Given at Goutelas, France, pp. 285-329, Editions Frontieres, Paris, France, 1989.

[3] W. Loud, Periodic Solutions of Perturbed Second-Order Autonomous Equation, Memoirs of the American Mathematical Society no. 47, American Mathematical Society, Providence, RI, USA, 1964

[4] G. Hamel, "Über erzwungene Schwingungen bei endlichen Amplituden," Mathematische Annalen, vol. 86, no. 1-2, pp. 1-13, 1922.

[5] D. Núñez and P. J. Torres, "KAM dynamics and stabilization of a particle sliding over a periodically driven curve," Applied Mathematics Letters, vol. 20, no. 6, pp. 610-615, 2007.

[6] R. Ortega, "A forced pendulum equation with many periodic solutions," Rocky Mountain Journal of Mathematics, vol. 27, no. 3, pp. 861-876, 1997.

[7] J. Lei, X. Li, P. Yan, and M. Zhang, "Twist character of the least amplitude periodic solution of the forced pendulum," SIAM Journal on Mathematical Analysis, vol. 35, no. 4, pp. 844-867, 2004 . 
[8] J. Mawhin, "Seventy-five years of global analysis around the forced pendulum equation," in Proceedings of the Equadiff 9, Conference on Differential Equations and Their Applications, pp. 115-145, Brno, Czech Republic, August 1997.

[9] J. Leray and J. Schauder, "Topologie et quations fonctionnelles," Annales Scientifiques de l'École Normale Supérieure, vol. 51, no. 3, pp. 45-78, 1934.

[10] J. Llibre and R. Ortega, "On the families of periodic orbits of the Sitnikov problem," SIAM Journal on Applied Dynamical Systems, vol. 7, no. 2, pp. 561-576, 2008.

[11] R. Ortega and A. Rivera, "Global bifurcations from the center of mass in the Sitnikov problem," Discrete and Continuous Dynamical Systems Series B, vol. 14, no. 2, pp. 719-732, 2010.

[12] S. Krantz and H. Parks, The Implicit Function Theorem History, Theory and Applications, Birkhäuser, Boston, Mass, USA, 2002.

[13] E. Freire, A. Gasull, and A. Guillamon, "First derivative of the period function with applications," Journal of Differential Equations, vol. 204, no. 1, pp. 139-162, 2004.

[14] R. Ortega, "The twist coefficient of periodic solutions of a timedependent Newton's equation," Journal of Dynamics and Differential Equations, vol. 4, no. 4, pp. 651-665, 1992.

[15] J. Sotomayor, Licões de Equacões Diferenciais Ordinárias, Proyecto Euclides No 11, Instituto de Matemática Pura e Aplicada (IMPA-CNPq), Rio de Janerio, Brasil, 1979.

[16] E. A. Coddington and N. Levinson, Theory of Ordinary Differential Equations, McGraw-Hill, New York, NY, USA, 1955.

[17] J. Möser, "On the invariant curves of area preserving mappings of an annulus," Nachrichten der Akademie der Wissenschaften in Göttingen. II. Mathematisch-Physikalische Klasse, vol. 2, pp. 120, 1962.

[18] R. Ortega, "Periodic solutions of a newtonian equation: stability by the third approximation," Journal of Differential Equations, vol. 128, no. 2, pp. 491-518, 1996.

[19] M. Zhang, "The best bound on the rotations in the stability of periodic solutions of a Newtonian equation," Journal of the London Mathematical Society, vol. 67, no. 1, pp. 137-148, 2003.

[20] D. Núñez and P. J. Torres, "On the motion of an oscillator with a periodically time-varying mass," Nonlinear Analysis: Real World Applications, vol. 10, no. 4, pp. 1976-1983, 2009.

[21] D. Núñez and P. J. Torres, "Stabilization by vertical vibrations," Mathematical Methods in the Applied Sciences, vol. 32, no. 9, pp. 1118-1128, 2009.

[22] H. Hanßmann and C. Simó, "Dynamical stability of quasi-periodic response solutions in planar conservative systems," Indagationes Mathematicae, vol. 23, no. 3, pp. 151-166, 2012. 


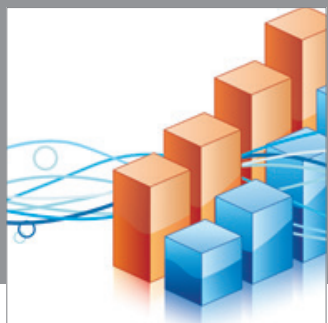

Advances in

Operations Research

mansans

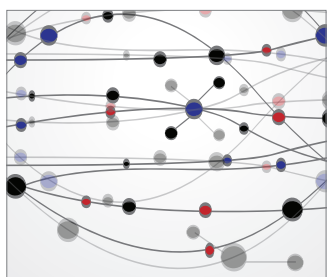

The Scientific World Journal
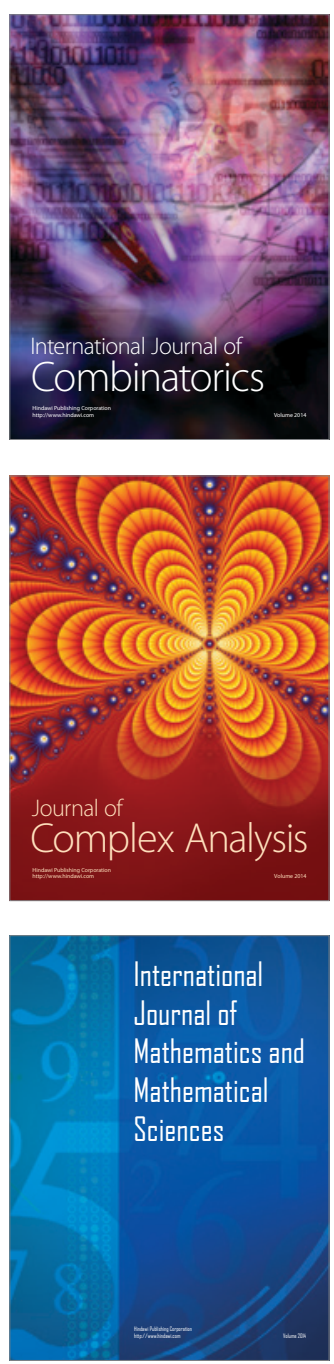
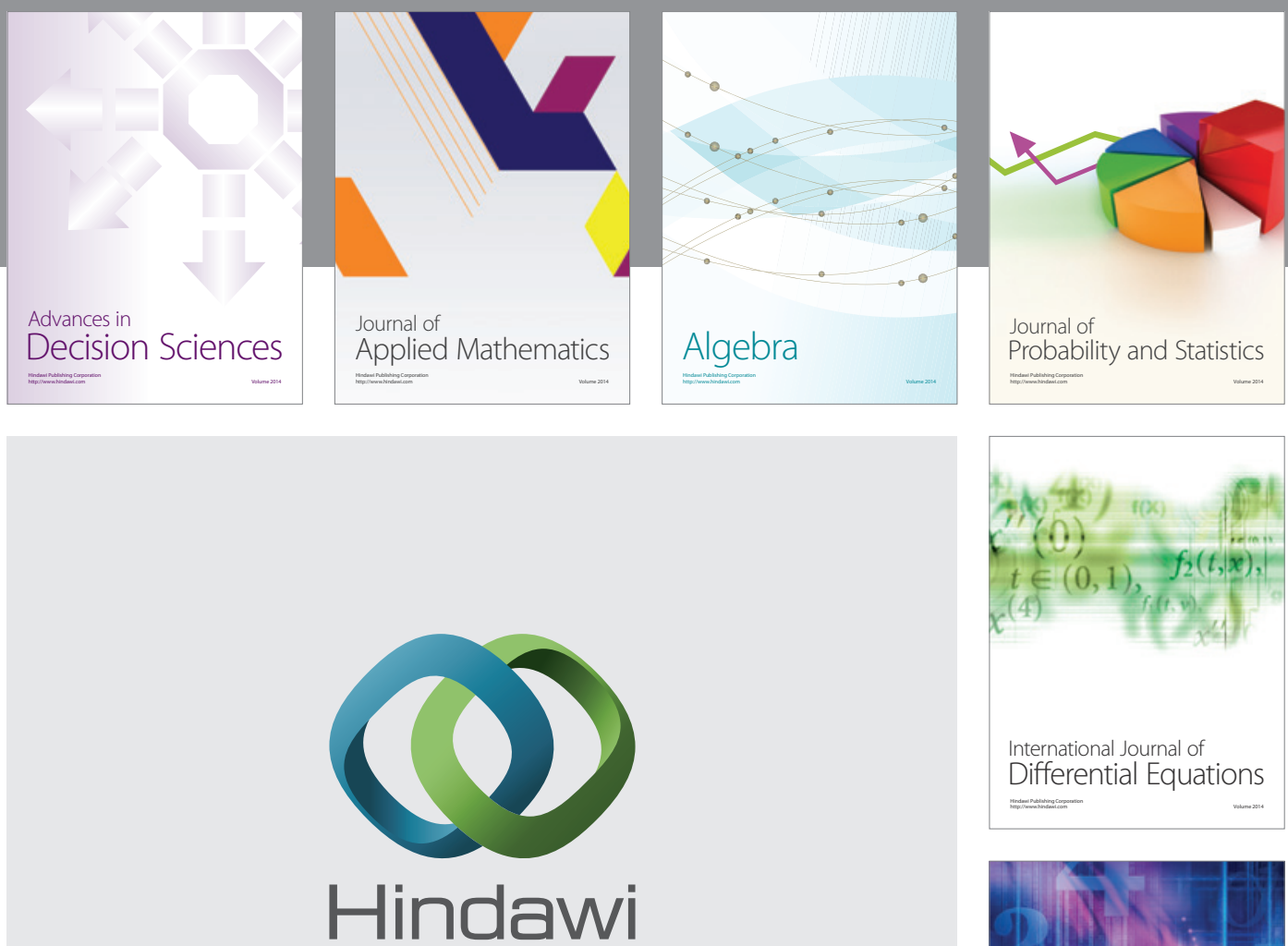

Submit your manuscripts at http://www.hindawi.com
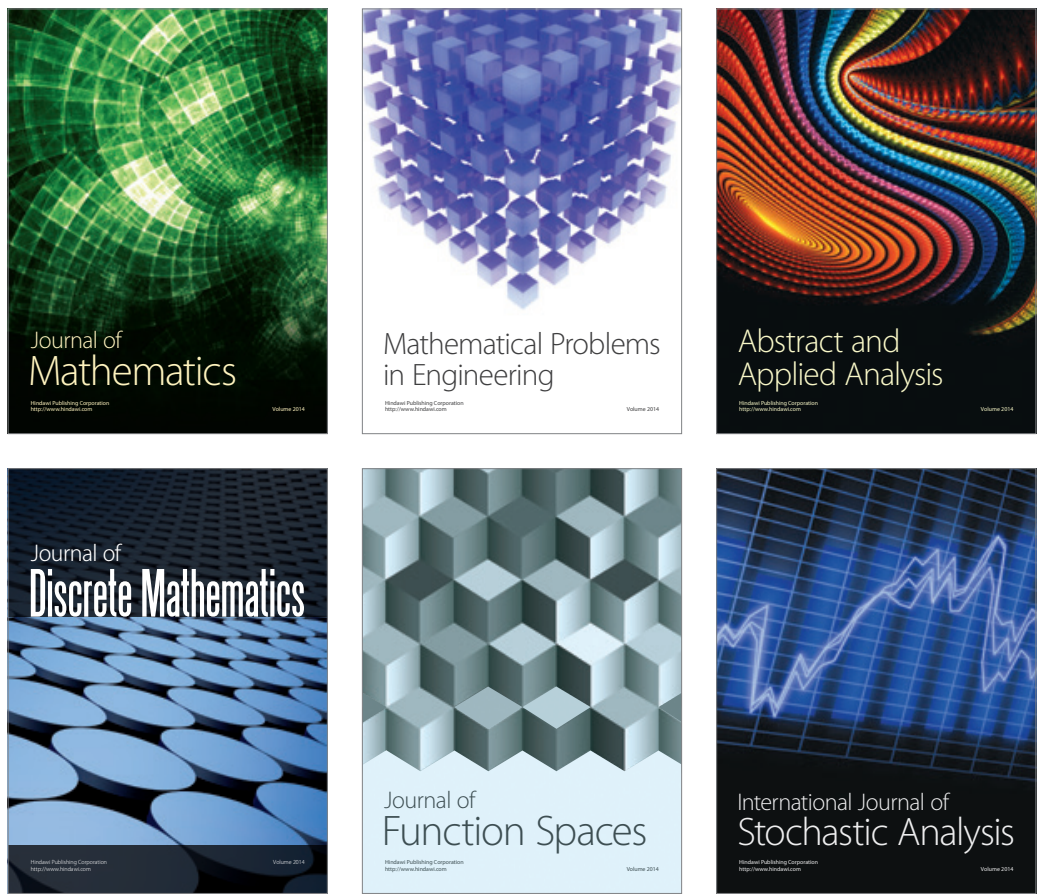

Journal of

Function Spaces

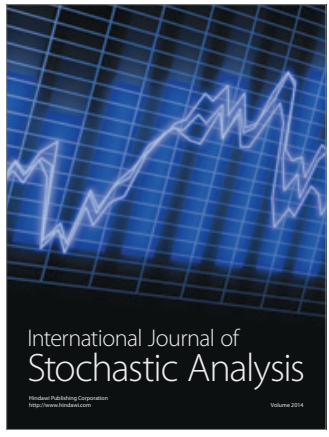

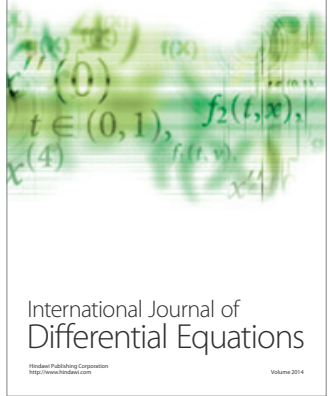
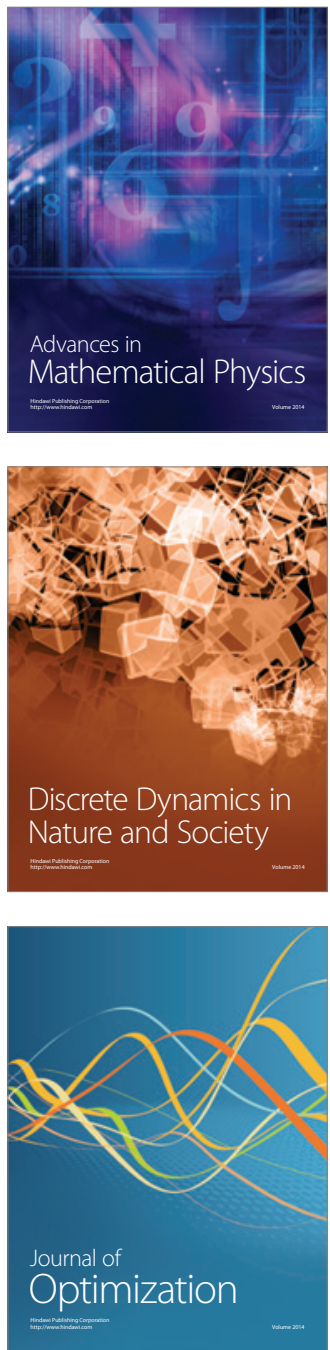